\title{
Effects of rhenium on graphene grown on $\mathrm{SiC}(0001)$
}

Chao Xia, Alexey Tal, Leif I Johansson, Weine Olovsson, Igor Abrikosov and Chariya Jacobi

The self-archived postprint version of this journal article is available at Linköping University Institutional Repository (DiVA):

http://urn.kb.se/resolve?urn=urn:nbn:se:liu:diva-145152

N.B.: When citing this work, cite the original publication.

Xia, C., Tal, A., Johansson, L. I, Olovsson, W., Abrikosov, I., Jacobi, C., (2018), Effects of rhenium on graphene grown on SiC(0oo1), Journal of Electron Spectroscopy and Related Phenomena, 222, 117121. https://doi.org/10.1016/j.elspec.2017.07.006

Original publication available at:

https://doi.org/10.1016/j.elspec.2017.07.006

Copyright: Elsevier

http://www.elsevier.com/

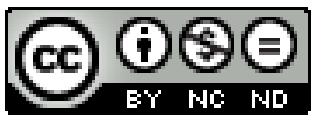




\title{
Effects of Rhenium on graphene grown on $\mathrm{SiC}(0001)$
}

\author{
Chao Xia ${ }^{1}$, Alexey A. Tal 1,2,*, Leif I. Johansson ${ }^{1}$, Weine Olovsson ${ }^{1}$, Igor A. Abrikosov ${ }^{1,2}$, \\ Chariya Jacobi ${ }^{1}$
}

1 Department of Physics, Chemistry, and Biology (IFM), Linköping University, S-58183 Linköping, Sweden

2 Materials Modeling and Development Laboratory, National University of Science and Technology “MISIS,” 119049 Moscow, Russia

* Correspondence: Electronic mail: aleta@ifm.liu.se (Alexey Tal).

\begin{abstract}
We study the effects of Rhenium (Re) deposited on epitaxial monolayer graphene grown on SiC(0001) and after subsequent annealing at different temperatures, by performing high resolution photoelectron spectroscopy (PES) and angle resolved photoelectron spectroscopy (ARPES). The graphene-Re system is found to be thermally stable. While no intercalation or chemical reaction of the Re is detected after deposition and subsequent annealing up to $1200^{\circ} \mathrm{C}$, a gradual decrease in the binding energy of the Re $4 \mathrm{f}$ doublet is observed. We propose that a larger mobility of the Re atoms with increasing annealing temperature and hopping of Re atoms between different defective sites on the graphene sample could induce this decrease of Re $4 \mathrm{f}$ binding energy. This is corroborated by first principles density functional theory (DFT) calculations of the Re core-level binding energy shift. No change in the doping or splitting of the initial monolayer graphene electronic band structure is observed after Re deposition and annealing up to $1200^{\circ} \mathrm{C}$, only a broadening of the bands.
\end{abstract}

Keywords: Rhenium, Graphene, photoelectron spectroscopy, core-level shift, ab initio density functional theory 


\section{Introduction}

The superb electronic and mechanical properties [1], [2] of graphene have attracted a tremendous interest in the past decade. Epitaxial graphene films grown on silicon carbide (SiC) substrates are considered as a potential solution for direct integration of graphene into electronic devices on a semiinsulating substrate, which avoids the hazardous transfer process. Furthermore, the use of SiC provides a potential for electronic applications operating at high frequencies, temperatures and voltages. However, the formation of defects during the growth process is unavoidable. The presence of commonly observed defects such as single vacancy, di-vacancy, and Stone-Wales point defect [3], [4], will not only induce a change of local configuration and curvature, but also affect the local density of $\pi$-electrons [5], [6] and enhance the reactivity of graphene [7]. It is also of high interest to obtain more knowledge about the local defects of graphene grown on $\mathrm{SiC}$ substrate.

The thermal stability for a few commonly used contact materials, such as gold (Au) [8], aluminum (Al) [9] and platinum (Pt) [10], have been tested for graphene grown on $\mathrm{SiC}$. $\mathrm{Au}, \mathrm{Al}$ and $\mathrm{Pt}$ are all found to intercalate into the graphene/SiC interface region after annealing at $800^{\circ} \mathrm{C}, 400^{\circ} \mathrm{C}$ and $800^{\circ} \mathrm{C}$, respectively. A metal with a higher melting point than Pt may be able to endure higher temperatures as a contact material for graphene. It is advantageous if a contact material for graphene does not form carbide, therefore rhenium can be a good candidate since all the other refractory metals niobium, molybdenum, tantalum, and tungsten are found to form carbides [11], [12]. Moreover, Re has a high resistance to poisoning induced by sulfuric and hydrochloric acids as well as corrosion by hydrogen, nitrogen, sulfur, and phosphorus. Re coatings are applied in high-temperature rocket engines to reduce wear and arc erosion [13]. Therefore, detailed investigations of the influence induced on the electronic structure and chemical composition by Re deposited on epitaxial graphene on Si-face SiC, and after subsequent annealing at different temperatures were carried out. Re serves perfectly as a contact material for graphene grown on SiC. No chemical reaction with the sample or intercalation of Re into the interface between the buffer layer and the SiC substrate is observed to take place after annealing up to $1200^{\circ} \mathrm{C}$. Further, no modification of the graphene electronic band structure, such as change in the doping or band splitting, is found to be induced by Re. For other works in the literature considering Re on graphene, see for example Refs. [28], [29], [30]. 


\section{Experimental}

\subsection{Sample preparation}

The samples used in the photoelectron spectroscopy (PES) and Angle resolved photoemission (ARPES) experiments are n-type nominally on-axis $6 \mathrm{H}-\mathrm{SiC}(0001)$ and $4 \mathrm{H}-\mathrm{SiC}(0001)$ substrates, respectively. The $6 \mathrm{H}-\mathrm{SiC}(0001)$ and respectively $4 \mathrm{H}-\mathrm{SiC}(0001)$ substrate has a misorientation error within $0.25^{\circ}$ and $0.05^{\circ}$. Both substrates were purchased from SiCrystal, with the Si face mechanically and chemically polished. RCA cleaning and HF etching of the substrates were applied to remove contaminations and surface oxides. Monolayer graphene was prepared by direct current heating of the substrates at a temperature of $\sim 1300^{\circ} \mathrm{C}$ for a few minutes, in a pressure of about $2 \times 10^{-7}$ torr. An electron beam evaporator, operated at its highest possible power, was used to evaporate Re onto the sample, kept at room temperature. The amount of Re obtained after several hours of deposition was estimated to be $\sim 0.3 \AA$, which corresponds to a coverage of $10-20 \%$.

\subsection{Characterization}

The characterizations were performed at beamlines I311 and I4 at the MAX-laboratory. Beamline I311 is equipped with a modified SX-700 monochromator and a large hemispherical Scienta electron analyzer, which allowed high resolution PES studies of the C 1s, Si 2p and Re 4f core levels to be carried out. The measured intensity ratios of the core levels allowed the amount of Re deposited on the sample to be estimated using a simple layer attenuation model. A total energy resolution of $<10$ to 100 $\mathrm{meV}$ at photon energies from 33 to $450 \mathrm{eV}$ and of $<300 \mathrm{meV}$ at energies from 600 to $750 \mathrm{eV}$ was then utilized. Beamline I4 is equipped with a spherical grating monochromator (SGM) and a PHOIBOS 100 2D CCD Specs electron energy analyzer. ARPES studies of in particular the $\pi$-band structure around the K-point in the Brillouin zone were carried out at this beamline, using the low angular dispersion (LAD) lens mode which provide an acceptant angle of $\pm 7^{\circ}$. The base pressure was about $1 \times 10^{-10} \mathrm{mbar}$ in both end-stations used.

\subsection{Computational details}


The calculations were performed within density functional theory (DFT), using the projector augmented-wave (PAW) method [14], [15] implemented in the Vienna ab initio simulation package (VASP)[16]. We employed the generalized gradient approximation (GGA) with the Perdew-BurkeErnzerhof (PBE) [17] exchange-correlation functional. The plane wave basis set cutoff energy was set to $400 \mathrm{eV}$. The Brillouin zone was sampled using a $5 \times 5 \times 1 \mathrm{k}$-point mesh.

The simulation box consists of $98 \mathrm{C}$ atoms for the defect-free graphene sheet. It was modeled using the $2.46 \times 2.46 \times 6.71 \AA$ graphene primitive cell, repeated by $7 \times 7 \times 1$ in the corresponding directions The optimization of the structures was performed with the convergence force $5 \times 10^{-4} \mathrm{eV} / \AA$. The size of the simulation box was constrained during the optimization.

In order to estimate the intercalation barriers, the nudged elastic band (NEB) [18] method calculation was performed. The intercalation barriers were found to be: for Re through Stone-Wales defect, $4.4 \mathrm{eV}$, single vacancy, $4.0 \mathrm{eV}$, and di-vacancy, $2.8 \mathrm{eV}$. The barriers were calculated on freestanding graphene. Therefore, penetration of Re atoms through the graphene layer resulting in intercalation between the graphene and the carbon buffer layer appears very unlikely in the temperature range the samples were heated. In order to understand how defects in a graphene layer affect core-levels of rhenium atoms, we performed ab initio DFT calculations of the core-level binding energy shifts (CLS) [19]. We assume that the core-hole at the ionized atom effectively acts as an extra proton. This assumption allows us to substitute the ionized atom of atomic number $\mathrm{Z}$ with the next element in the Periodic Table. This approximation is also called an equivalent core or $(\mathrm{Z}+1)$-approximation [20,21]. It is known to be reliable and to reproduce experiment well [22].

\section{Results and discussion}

High-resolution core level experiments were performed before and after Re deposition and also after subsequent annealing at different temperatures. A set of C 1s spectra collected using a photon energy of $600 \mathrm{eV}$ are displayed in Fig. 1(a). For the as-prepared monolayer graphene sample (bottom spectrum) the $\mathrm{C}$ 1s spectrum is composed of three components, labeled $\mathrm{B}, \mathrm{G}$ and $\mathrm{SiC}$, that correspond to carbon buffer layer, graphene, and SiC substrate, respectively. From the extracted G/SiC and G/B intensity ratios a graphene layer thickness of $\sim 1 \mathrm{ML}$ is estimated [23]. No obvious changes in the $\mathrm{C} 1 \mathrm{~s}$ spectrum 
are observed after Re deposition and after subsequent annealing at temperatures up to $1200^{\circ} \mathrm{C}$. Only a small increase of the energy separation between $\mathrm{G}$ peak and SiC peak, by $\sim 0.12 \mathrm{eV}$, is observed after annealing at $1200^{\circ} \mathrm{C}$ compared to the initial sample. This value is much smaller compared to the binding energy shifts induced upon intercalation at the interface of elements such as Li [24] and $\mathrm{Na}$ [25]. The G/B intensity ratio is found to increase slightly, by $\sim 10 \%$, after annealing at $1200^{\circ} \mathrm{C}$. The possibility that Re has intercalated into the $\mathrm{SiC}$ substrate interface can therefore be ruled out since much larger $\mathrm{G} / \mathrm{B}$ and $\mathrm{G} / \mathrm{SiC}$ intensity ratios would be expected, as well as a larger energy separation between the $\mathrm{G}$ and $\mathrm{SiC}$ peaks. Instead, the observed changes can indicate that $\mathrm{Re}$ atoms have penetrated through the graphene layer and reside on the buffer layer since this would increase the G/B intensity ratio.

A series of Si 2p core level spectra recorded using a photon energy of $190 \mathrm{eV}$ before and after Re deposition, as well as after subsequent annealing, are illustrated in Fig. 1(b). They exhibit quite similar trends as the C 1s spectra shown in Fig. 1(a). The Si 2p spectrum of the initial 1ML graphene sample
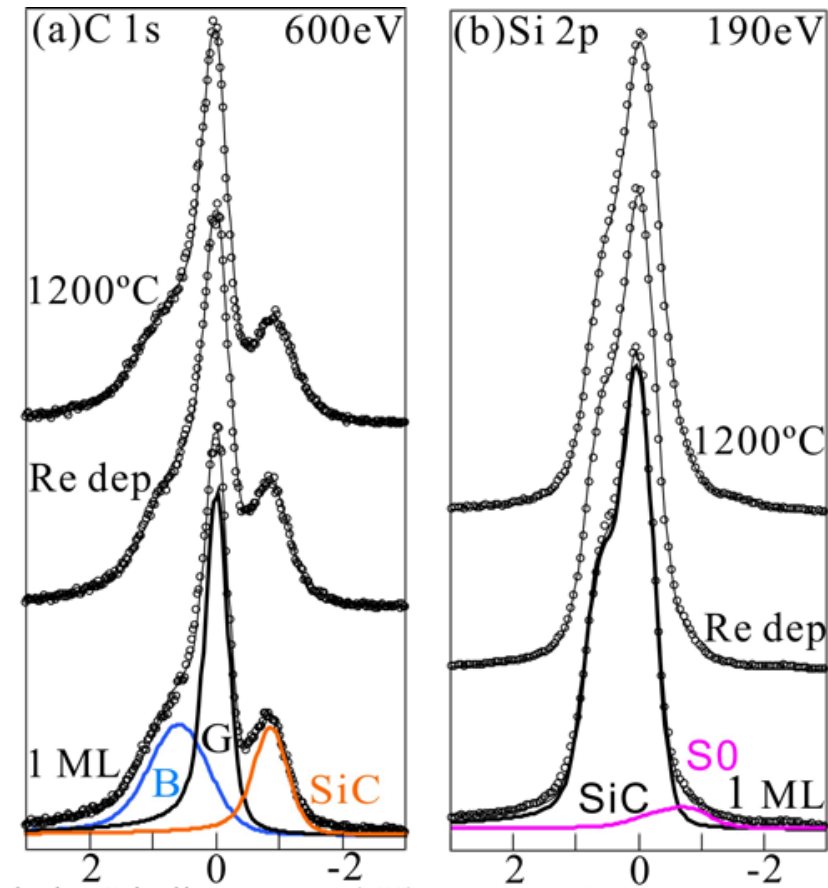

Relative Binding energy (eV)Relative Binding energy (eV) 
Figure 1. (a) C 1s and (b) Si 2p core level spectra collected using a photon energy of $600 \mathrm{eV}$ and 190 $\mathrm{eV}$, respectively, from the initial in-situ prepared $1 \mathrm{ML}$ graphene sample, after Re deposition and after subsequent annealing at $1200^{\circ} \mathrm{C}$.

comprises two spin-split Si 2p doublets. The bulk SiC substrate component, labeled SiC, dominates but another minor component is discernable, labeled $\mathrm{S} 0$ and shifted $0.8 \mathrm{eV}$ to lower binding energy. This component may originate from Si clusters or defects formed at the interface region. No significant changes, such as appearance of additional shifted peaks or peak broadening, are observed in the Si $2 \mathrm{p}$ spectrum after Re deposition and subsequent annealing up to $1200^{\circ} \mathrm{C}$. This indicates that no Re atoms are able to intercalate into the SiC substrate interface and react with Si.

The Re 4f core level spectra recorded after Re deposition and subsequent annealing are shown in Fig. 2(a). One Re $4 \mathrm{f}$ doublet, labeled $\mathrm{Re}$, is observed after Re deposition. The $4 \mathrm{f}_{7 / 2}$ component is determined to be located at a binding energy of $41.0 \mathrm{eV}$, using a curve fit procedure [26]. Surprisingly, the Re peak is found to shift continuously towards lower binding energy with increasing annealing temperature, without any substantial change in the width (FWHM value) of the Re $4 \mathrm{f}$ peak. We have repeated the experiment three times in order to rule out any experimental error or random event. The same shift in binding energy, within an error bar of $\pm 0.05 \mathrm{eV}$ of the $\operatorname{Re} 4 \mathrm{f}_{7 / 2}(\Delta \mathrm{BE})$ component versus annealing temperature was obtained each time, and is displayed in Fig. 2(b). The total shift of the Re $4 \mathrm{f}$ level after annealing to $1200^{\circ} \mathrm{C}$ is as large as $0.61 \mathrm{eV}$. To the best of our knowledge, such phenomenon, without being related with simultaneous intercalation into the graphene/SiC interface or chemical reaction processes, was not reported earlier. Based on the theoretical calculation reported below, we speculate that the shift of the binding energy of the Re $4 \mathrm{f}$ level is related to the hopping of Re atoms between different defective sites, such as vacancy, grain boundary, Stone-Wales defects on the graphene and carbon buffer layer. One thing needs to be addressed: the coverage of Re in our experiment is only $10-20 \%$ due to the difficulty of evaporating Re. Moreover, that in-situ grown graphene samples are used which contain much smaller graphene grains and consequently also a higher density of defective sites than $e x$-situ grown samples. The total number of Re atoms may therefore be considerably less compared to the number of defective sites available on our as prepared samples. If the coverage of Re would be much larger, multiple peaks or broader peaks are expected to be present in Re 
4f spectrum, because the amount of Re atoms would then oversaturate the number of different defective sites, and therefore more than one type of defective site must be occupied by Re atoms simultaneously.
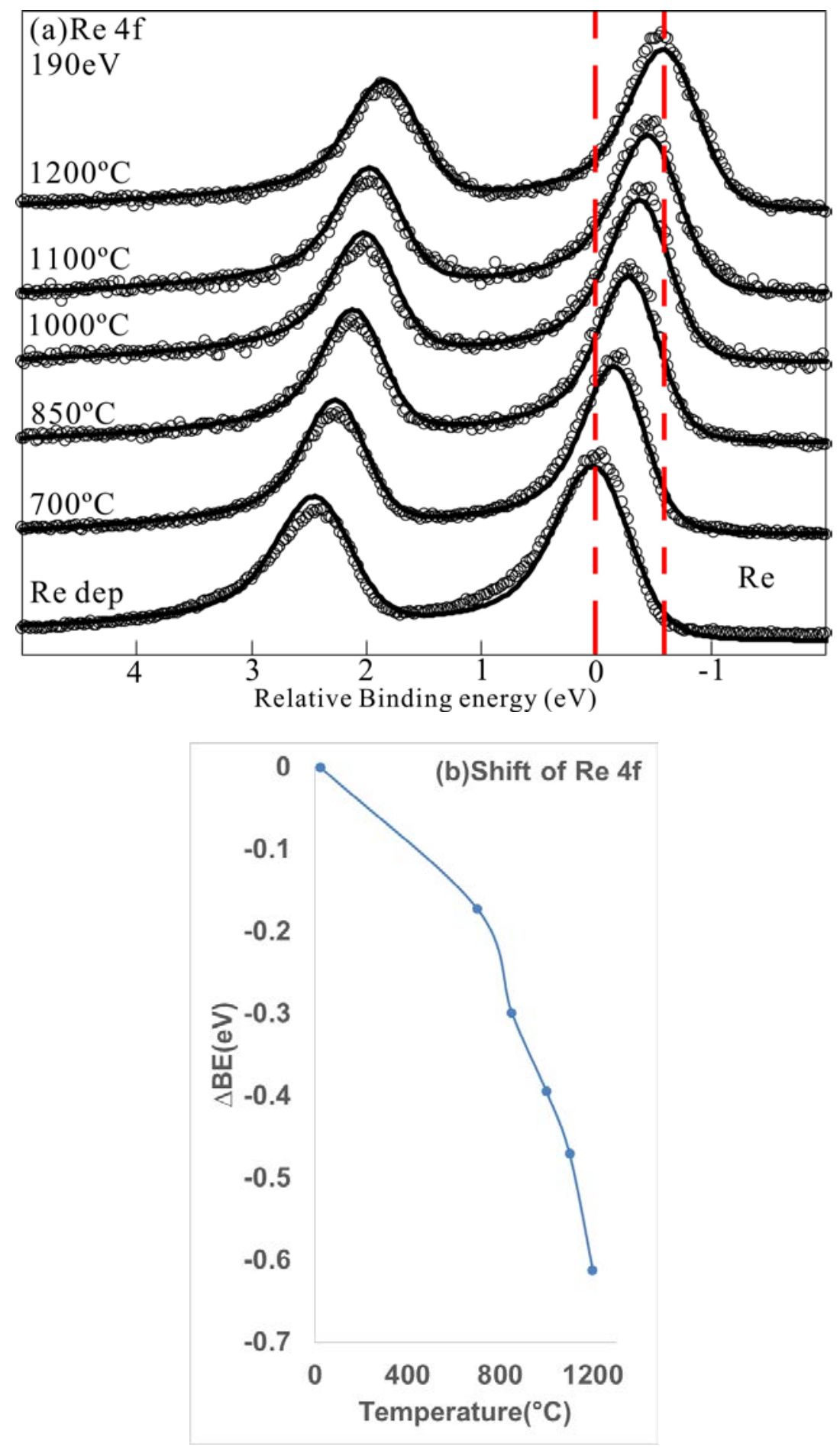
Figure 2. (a) Re 4f core level spectra acquired at a photon energy of $190 \mathrm{eV}$ after Re deposition and subsequent annealing at different temperatures and (b) shift of Re 4f binding energy with annealing temperature. The shifts are determined within $\pm 0.05 \mathrm{eV}$, and the line is just a guide to the eye.

ARPES is a powerful tool for investigating changes induced in the electronic band structure. The $\pi$ band structure close to the $\mathrm{K}$ point of the graphene Brillouin zone was studied after Re deposition and subsequent annealing, using a photon energy of $33 \mathrm{eV}$. The initial $1 \mathrm{ML}$ graphene sample shows one linearly dispersing $\pi$-band with

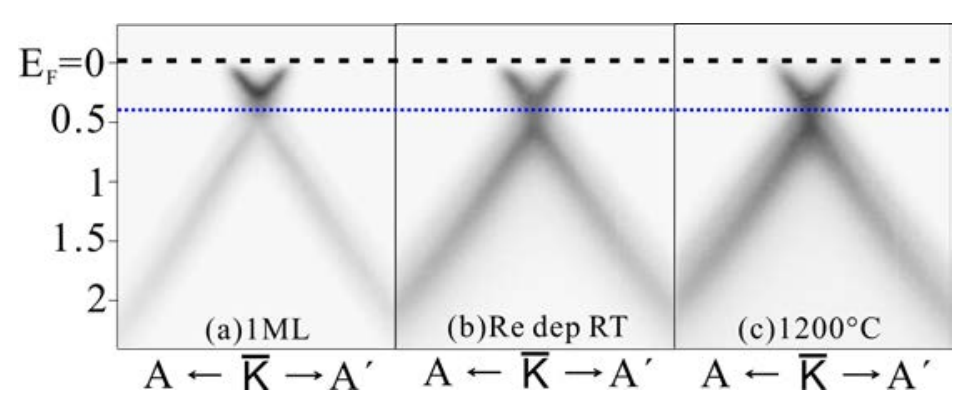

Figure 3. The $\pi$-band dispersion recorded around the K-point, using a photon energy of $33 \mathrm{eV}$, from (a) the initial $1 \mathrm{ML}$ graphene sample, (b) after Re deposition at room temperature and (c) after subsequent annealing at temperatures of $1200^{\circ} \mathrm{C}$.

the Dirac point located at $\sim 0.4 \mathrm{eV}$ below the Fermi level [27], as displayed in Fig. 3(a). A more diffuse $\pi$-band, Fig. 3(b), is observed after Re deposition. The local scattering induced by the deposited Re atoms is suggested to be the reason. Annealing up to $1200^{\circ} \mathrm{C}$ induces no additional changes except a slight broadening of the $\pi$-band, see Fig. 4(c). This broadening is attributed to local disturbances induced by the deposited Re. If the Re atoms upon heating, stay on the surface or penetrate through the graphene and reside on the carbon buffer layer, is not possible to determine from this slight broadening. The Dirac point remains at the same location after Re deposition and subsequent annealing cycles, which implies that no additional doping is induced into the system. 
Theoretical calculations were carried out for the three most common graphene defects: vacancy, divacancy and Stone-Wales (SW) defect, performed with a Re atom in vicinity of these defects. In all simulations, the structure was fully relaxed after placing the rhenium atom on top of the graphene layer. A vacancy defect is a very favorable spot for the Re atom. The total energy difference of the configurations, where the Re atom is sitting next to a vacancy (Fig.4 (a)) and in a vacancy (Fig.4(b)), is $8.18 \mathrm{eV}$. From Fig. 4 (b) it is seen that the rhenium atom causes noticeable out-of-plane distortions in the graphene layer. In particular, it pulls out three carbon atoms, which become its nearest
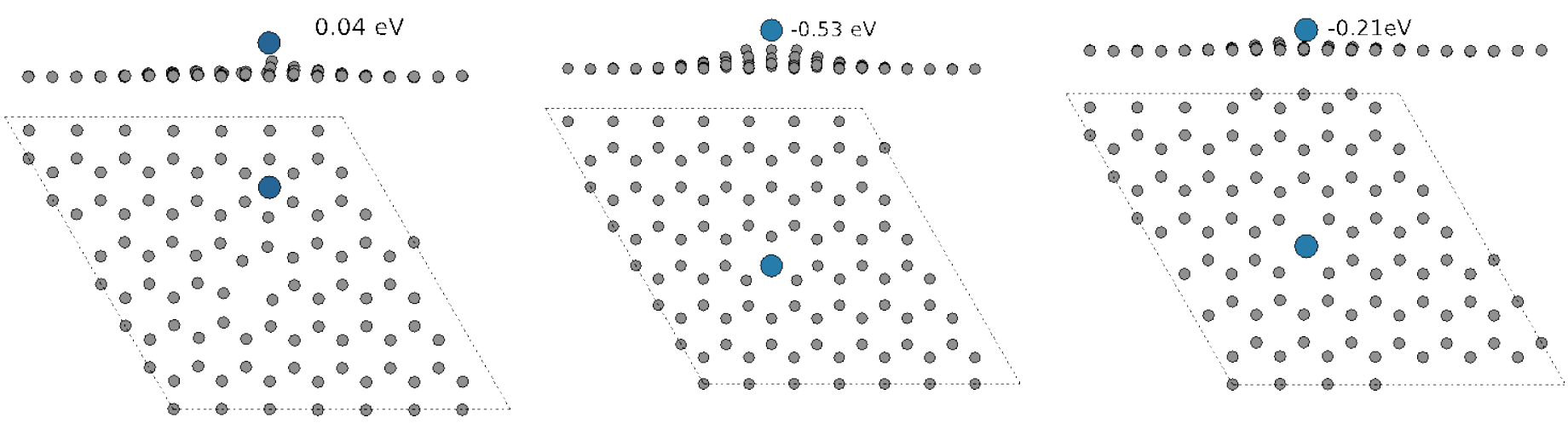

Figure 4. Local structure and core-level shift calculated for a rhenium atom located at different positions; (a) in a 6-chain ring next to a vacancy, (b) in a vacancy and (c) in a di-vacancy.

neighbors. This reconstruction results in a shift of the rhenium core-level by $-0.53 \mathrm{eV}$. In case of a divacancy the rhenium atom has four carbon atoms in the first coordination shell and does not induce such big distortions (Fig.4(c)). Here, Re atoms sitting in the di-vacancy defect exhibits a core-level shift of $-0.21 \mathrm{eV}$. From these results, we conclude that defects that give a lower coordination for Re atoms produces a negative CLS.

SW defects are formed by an in-plane $90^{\circ}$ rotation of two carbon atoms with respect to the midpoint of the bond. In this transformation four hexagons are changed into two heptagons and two pentagons. Heptagons have a lower density of atoms compared to a regular graphene hexagon, which makes the SW defect a possible candidate for causing negative CLS in rhenium. Our calculations showed that a rhenium atom trapped in a pentagon of a SW defect, as seen in Fig. 5 (a), corresponds to the lowest 
total energy of the three configurations considered in Fig. 5. This atomic configuration was obtained by relaxing the structure, after the rhenium atom was
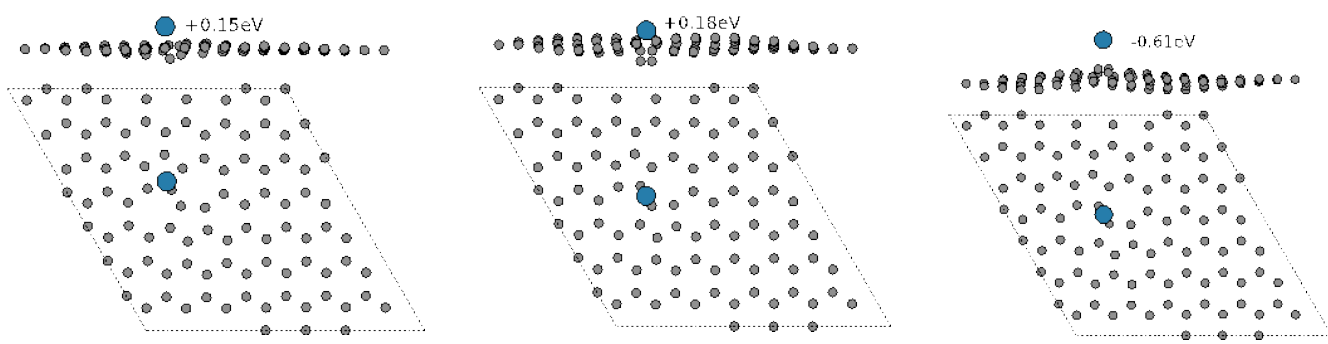

Figure 5. (a) Local structure and core-level shifts calculated for rhenium atom sitting in a 5-chain ring of SW defect; (b) in a 7-chain ring of SW defect; (c) in a 7-chain ring of SW defect and in metastable configuration.

placed right above the center of the pentagon in the graphene layer. Structural relaxation led to that a carbon atom is pushed out of the plane, thus forming a well under the rhenium atom. The CLS of the Re atom in this well is $+0.15 \mathrm{eV}$.

Another configuration shown in Fig. 5 (b) was obtained after placing the Re atom in the center of a heptagon and relaxing the structure. The rhenium atom then pushed two carbon atoms out of the plane and occupied the formed well. The rhenium CLS for this configuration is $+0.18 \mathrm{eV}$ but the total energy is $0.19 \mathrm{eV}$ higher than in the former case. The configuration in Fig. 5 (c) is particularly interesting. It represents a metastable state which has a $1.1 \mathrm{eV}$ higher total energy than the pentagon case in Fig.5 (a). It was obtained after placing the rhenium atom on a graphene layer that has a wave structure. In this configuration the rhenium atom is sitting on a wave crest and thus has only two nearest neighbors which results in a negative CLS of $-0.61 \mathrm{eV}$. It is also worth mentioning that in real systems the presence of a substrate beneath the graphene layer, may favor the configuration shown in Fig. 5 (c).

\section{Conclusion}

The effects induced in the electronic structure of monolayer graphene, epitaxially grown on SiC(0001), after Re deposition and after subsequent annealings are investigated in detail using PES and ARPES. 
Very limited changes in the electronic properties of monolayer graphene are observed after Re deposition and after annealing at temperatures up to $1200^{\circ} \mathrm{C}$. The $\mathrm{C} 1 \mathrm{~s}$ and $\mathrm{Si} 2 \mathrm{p}$ core levels remain essentially unaffected after deposition and annealing, only a gradual decrease in the binding energy of the Re $4 \mathrm{f}$ doublet is observed with increasing annealing temperature. Thus, no intercalation of Re atoms into the graphene/SiC interface or reaction with the graphene is detected after Re deposition and annealing up to $1200^{\circ} \mathrm{C}$.

Our theoretical calculations have clearly shown that presence of defects in the graphene layer can be responsible for the observed negative binding energy shifts of the Re $4 \mathrm{f}$ levels. An increase in the temperature results in higher mobility of the Re atoms on the graphene surface, which will allow the Re atoms to occupy defective sites and thereby significantly minimizing the energy. Hence, we have serious reasons to assume that the negative CLS observed in experiments can be explained by the interaction with defects.

The single $\pi$-band structure observed from the initial sample is only found to broaden somewhat after Re deposition and after subsequent annealing up to $1200^{\circ} \mathrm{C}$. No shift or splitting of the band is revealed, which would in such case indicate additional doping or intercalation at the graphene/SiC interface. From the perspective that Re does not react with, or intercalates into the graphene/SiC interface to create a bilayer instead of monolayer graphene, rhenium constitutes a very suitable contact material for graphene based electronic device for high temperature applications.

\section{ACKNOWLEDGMENTS}

Alexey A. Tal, Weine Olovsson and Igor A. Abrikosov gratefully acknowledge the financial support of the Knut and Alice Wallenberg Foundation through Grant No. 2012.0083 and 2014-2019 (COTXS), the Swedish Foundation or Strategic Research (SSF) program SRL Grant No. 10-0026, the Swedish Research Council (VR) Grant No. 2015-04391, and the Swedish Government Strategic Research Area in Materials Science on Functional Materials at Linköping University (Faculty Grant SFO-Mat-LiU No. 2009-00971). Alexey A. Tal and Igor A. Abrikosov are grateful for support of the Ministry of Education and Science of the Russian Federation, Grant No. 14.Y26.31.0005 and in the framework of 
Increase Competitiveness Program of MISiS. C. Jacobi gratefully acknowledges support from the

Swedish Research Council \#621-2011-4252

\section{REFERENCES}

[1] A. K. Geim, “Graphene: status and prospects.,” Science, vol. 324, no. 5934, pp. 1530-4, Jun. 2009.

[2] C. Berger, Z. Song, X. Li, X. Wu, N. Brown, C. Naud, D. Mayou, T. Li, J. Hass, A. N. Marchenkov, E. H. Conrad, P. N. First, and W. A. de Heer, "Electronic confinement and coherence in patterned epitaxial graphene.,” Science, vol. 312, no. 5777, pp. 1191-6, May 2006.

[3] F. Banhart, J. Kotakoski, and A. V. Krasheninnikov, "Structural Defects in Graphene,” ACS Nano, vol. 5, no. 1, pp. 26-41, Jan. 2011.

[4] H. Terrones, R. Lv, M. Terrones, and M. S. Dresselhaus, "The role of defects and doping in 2D graphene sheets and 1D nanoribbons,” Reports Prog. Phys., vol. 75, p. 062501, 2012.

[5] X. Y. Peng and R. Ahuja, "Symmetry Breaking Induced Bandgap in Epitaxial Graphene Layers on SiC307,” Nano Lett., vol. 8, no. 12, pp. 4464-4468, 2008.

[6] D. W. Boukhvalov and M. I. Katsnelson, "Chemical Functionalization of Graphene with Defects,” Nano Lett., vol. 8, no. 12, pp. 4373-4379, Dec. 2008.

[7] E. J. Duplock, M. Scheffler, and P. J. D. Lindan, “Hallmark of Perfect Graphene,” Phys. Rev. Lett., vol. 92, no. 22, p. 225502, Jun. 2004.

[8] I. Gierz, T. Suzuki, R. T. Weitz, D. S. Lee, B. Krauss, C. Riedl, U. Starke, H. Höchst, J. H. Smet, C. R. Ast, and K. Kern, "Electronic decoupling of an epitaxial graphene monolayer by gold intercalation,” Phys. Rev. B, vol. 81, no. 23, p. 235408, Jun. 2010.

[9] C. Xia, L. I. Johansson, A. A. Zakharov, L. Hultman, and C. Virojanadara, "Effects of Al on epitaxial graphene grown on 6H-SiC(0001),” Mater. Res. Express, vol. 1, no. 1, p. 015606, Mar. 2014.

[10] C. Xia, L. I. Johansson, Y. Niu, A. A. Zakharov, E. Janzén, and C. Virojanadara, “High thermal stability quasi-free-standing bilayer graphene formed on $4 \mathrm{H}-\mathrm{SiC}(0001)$ via platinum intercalation,” Carbon., vol. 79, pp. 631-635, Nov. 2014.

[11] J. Diaz, “Pure rhenium metal,” Potentials, IEEE, no. March, pp. 37-39, 1996.

[12] A. Naor, N. Eliaz, E. Gileadi, and S. Taylor, "Properties and applications of rhenium and its alloys,” AMMTIAC Q., vol. 5, no. 1, pp. 11-15, 2010.

[13] B. D. Reed, J. a. Biaglow, and S. J. Schneider, "Engineering Issues of Iridium Coated Rhenium Rockets,” Mater. Manuf. Process., vol. 13, no. 5, pp. 757-771, Sep. 1998.

[14] G. Kresse and D. Joubert, "From ultrasoft pseudopotentials to the projector augmented-wave method,” Phys. Rev. B, vol. 59, no. 3, pp. 1758-1775, Jan. 1999.

[15] P. E. Blöchl, "Projector augmented-wave method,” Phys. Rev. B, vol. 50, no. 24, pp. 1795317979, Dec. 1994.

[16] J. P. Perdew, K. Burke, and M. Ernzerhof, “Generalized Gradient Approximation Made Simple,” Phys. Rev. Lett., vol. 77, no. 18, pp. 3865-3868, Oct. 1996. 
[17] G. Kresse and J. Furthmüller, "Efficient iterative schemes for ab initio total-energy calculations using a plane-wave basis set," Phys. Rev. B, vol. 54, no. 16, pp. 11169-11186, Oct. 1996.

[18] Henkelman G, Uberuaga BP, Jónsson H "A climbing image nudged elastic band method for finding saddle points and minimum energy paths". J Chem Phys, vol.113, no. 22, pp. 99019904, Oct. 2000

[19] W. Olovsson, T. Marten, E. Holmström, B. Johansson, and I. A. Abrikosov, "First principle calculations of core-level binding energy and Auger kinetic energy shifts in metallic solids," $J$. Electron Spectros. Relat. Phenomena, vol. 178-179, pp. 88-99, May 2010.

[20] Alberto Cavallin, Monica Pozzo, Cristina Africh, Alessandro Baraldi, Erik Vesselli, Carlo Dri, Giovanni Comelli, Rosanna Larciprete, Paolo Lacovig, Silvano Lizzit, and Dario Alfè, ”Local Electronic Structure and Density of Edge and Facet Atoms at Rh Nanoclusters Self-Assembled on a Graphene Template” ACS Nano, vol. 6, no. 4,pp. 3034-3043, 2012

[21] B. Johansson and N. Mårtensson, "Core-level binding-energy shifts for the metallic elements," Phys. Rev. B, vol. 21, no. 10, pp. 4427-4457, May 1980.

[22] T. Marten, W. Olovsson, S. I. Simak, and I. A. Abrikosov, "Ab initio study of disorder broadening of core photoemission spectra in random $\mathrm{Cu}-\mathrm{Pd}$ and $\mathrm{Ag}-\mathrm{Pd}$ alloys," Phys. Rev. B, vol. 72, no. 5, p. 054210, Aug. 2005.

[23] C. Virojanadara, M. Syväjarvi, R. Yakimova, L. I. Johansson, A. Zakharov, and T. Balasubramanian, "Homogeneous large-area graphene layer growth on $6 \mathrm{H}-\mathrm{SiC}(0001)$," Phys. Rev. B, vol. 78, no. 24, p. 245403, Dec. 2008.

[24] C. Virojanadara, S. Watcharinyanon, A. A. Zakharov, and L. I. Johansson, "Epitaxial graphene on 6H-SiC and Li intercalation,” Phys. Rev. B, vol. 82, no. 20, p. 205402, Nov. 2010.

[25] S. Watcharinyanon, L. I. Johansson, C. Xia, and C. Virojanadara, "Changes in structural and electronic properties of graphene grown on $6 \mathrm{H}-\mathrm{SiC}(0001)$ induced by Na deposition,” J. Appl. Phys., vol. 111, no. 8, p. 083711, 2012.

[26] "The parameters utilized for Re $4 \mathrm{f}$ spectra were a spin orbit splitting of $2.43 \mathrm{eV}$, a branching ratio of 0.75 , an asymmetry parameter of 0.18 and a Gaussian and a Lorentzian widths of $0.57 \pm 0.06 \mathrm{eV}$ and $0.15 \mathrm{eV}$ respectively.”

[27] T. Ohta, A. Bostwick, J. McChesney, T. Seyller, K. Horn, and E. Rotenberg, "Interlayer Interaction and Electronic Screening in Multilayer Graphene Investigated with Angle-Resolved Photoemission Spectroscopy,” Phys. Rev. Lett., vol. 98, no. 20, p. 206802, May 2007.

[28] C. Tonnoir, A. Kimouche, J. Coraux, L. Magaud, B. Delsol, B. Gilles, and C. Chapelier, "Induced Superconductivity in Graphene Grown on Rhenium”, Phys. Rev. Lett., vol. 111, p. 246805, 2013.

[29] O. Miramontes, F. Bonafé, U. Santiago, E. Larios-Rodriguez, J.J. Velazquez-Salazar, M.M. Mariscal, and M.J. Yacaman, "Ultra-small rhenium clusters supported on graphene”, Phys. Chem. Chem. Phys., vol. 17, p. 7898, 2015.

[30] F. Ferrante, A. Prestianni, R. Cortese, R. Schimmenti, and D. Duca, "Density Functional Theory Investigation on the Nucleation of Homo- and Heteronuclear Metal Clusters on Defective Graphene”, J. Phys. Chem. C, vol. 120, p. 12022, 2016. 


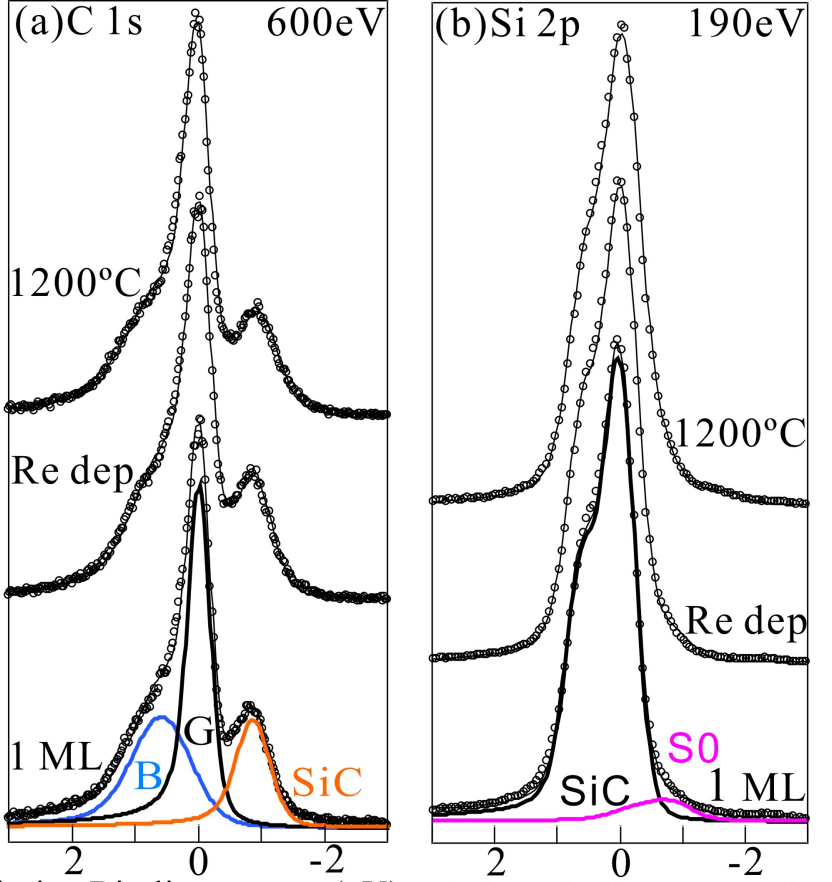

Relative Binding energy $(\mathrm{eV})$ Relative Binding energy $(\mathrm{eV})$ 


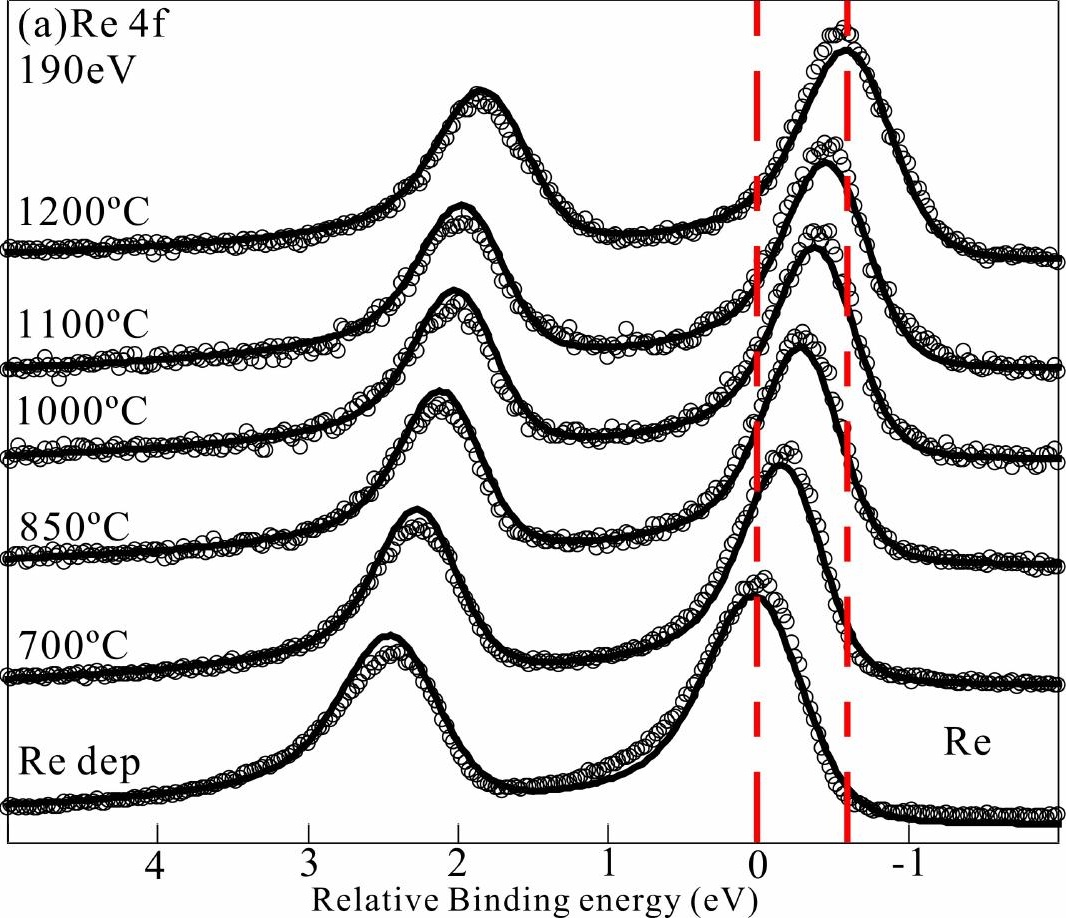




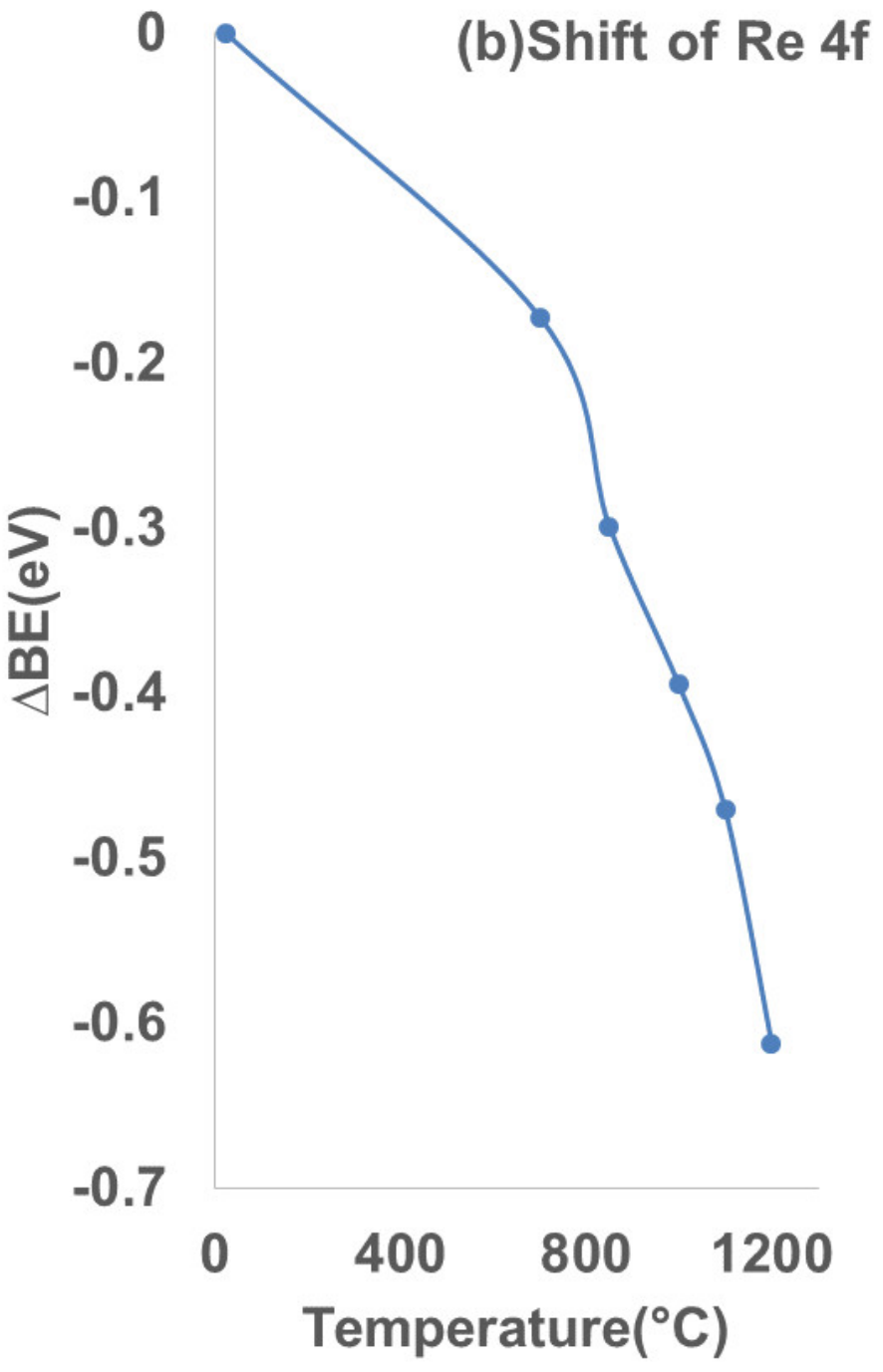




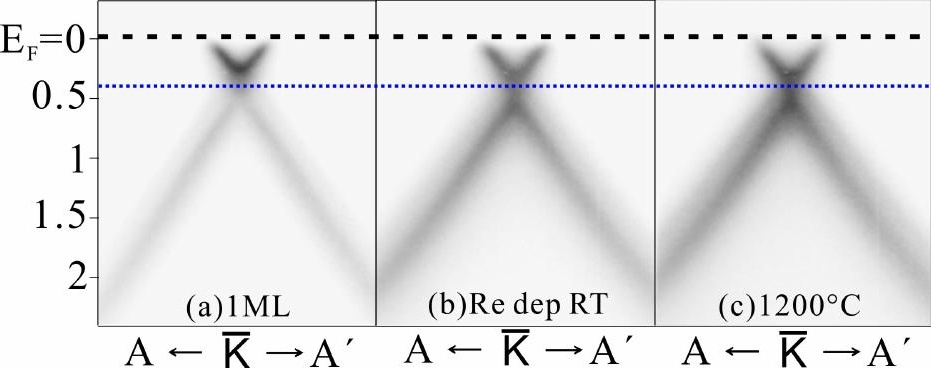




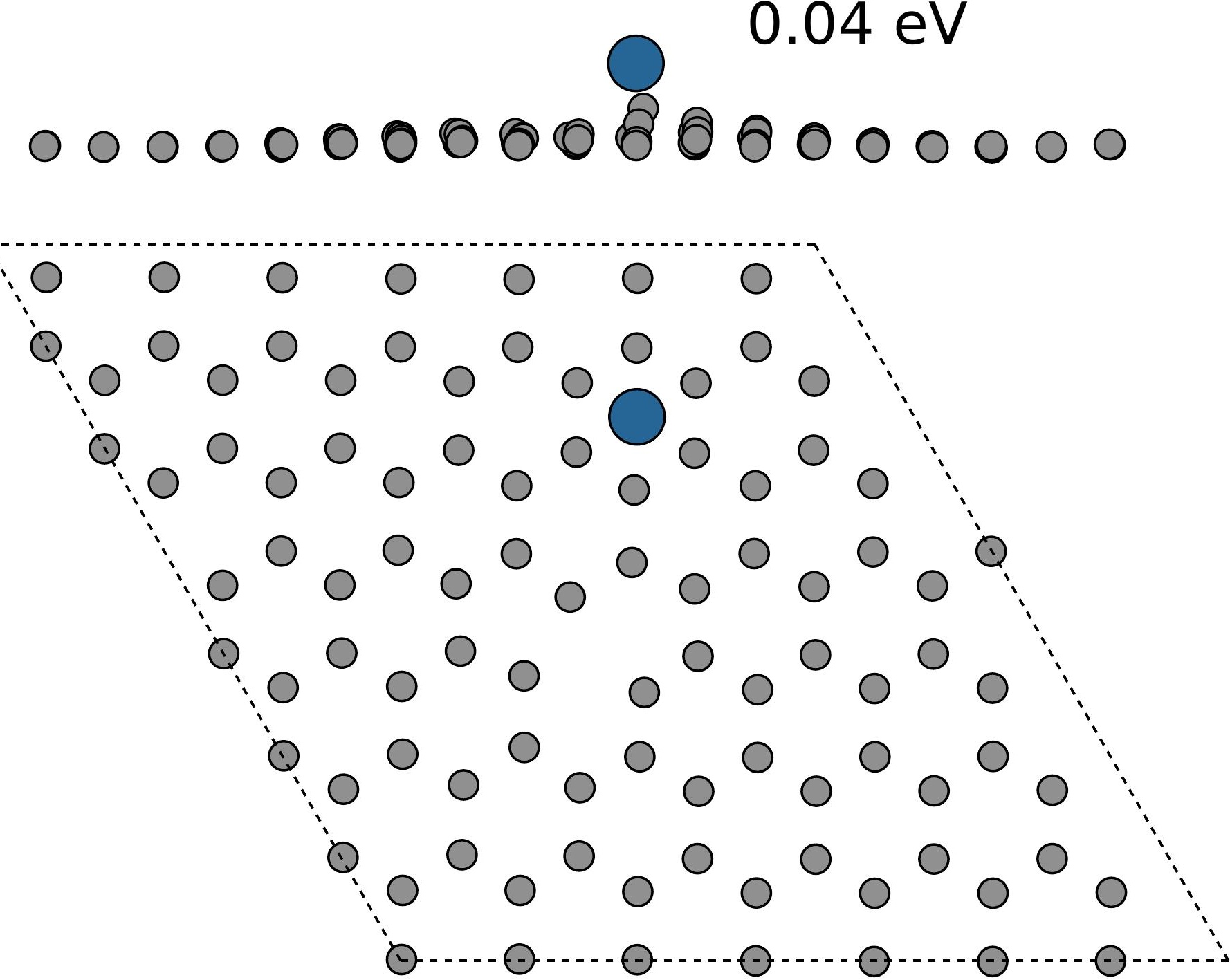




\section{$-0.53 \mathrm{eV}$}

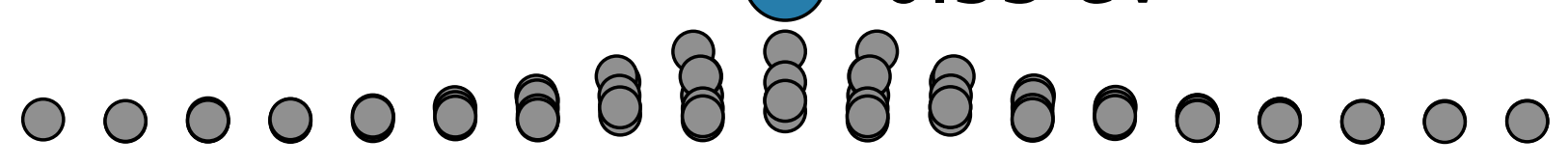

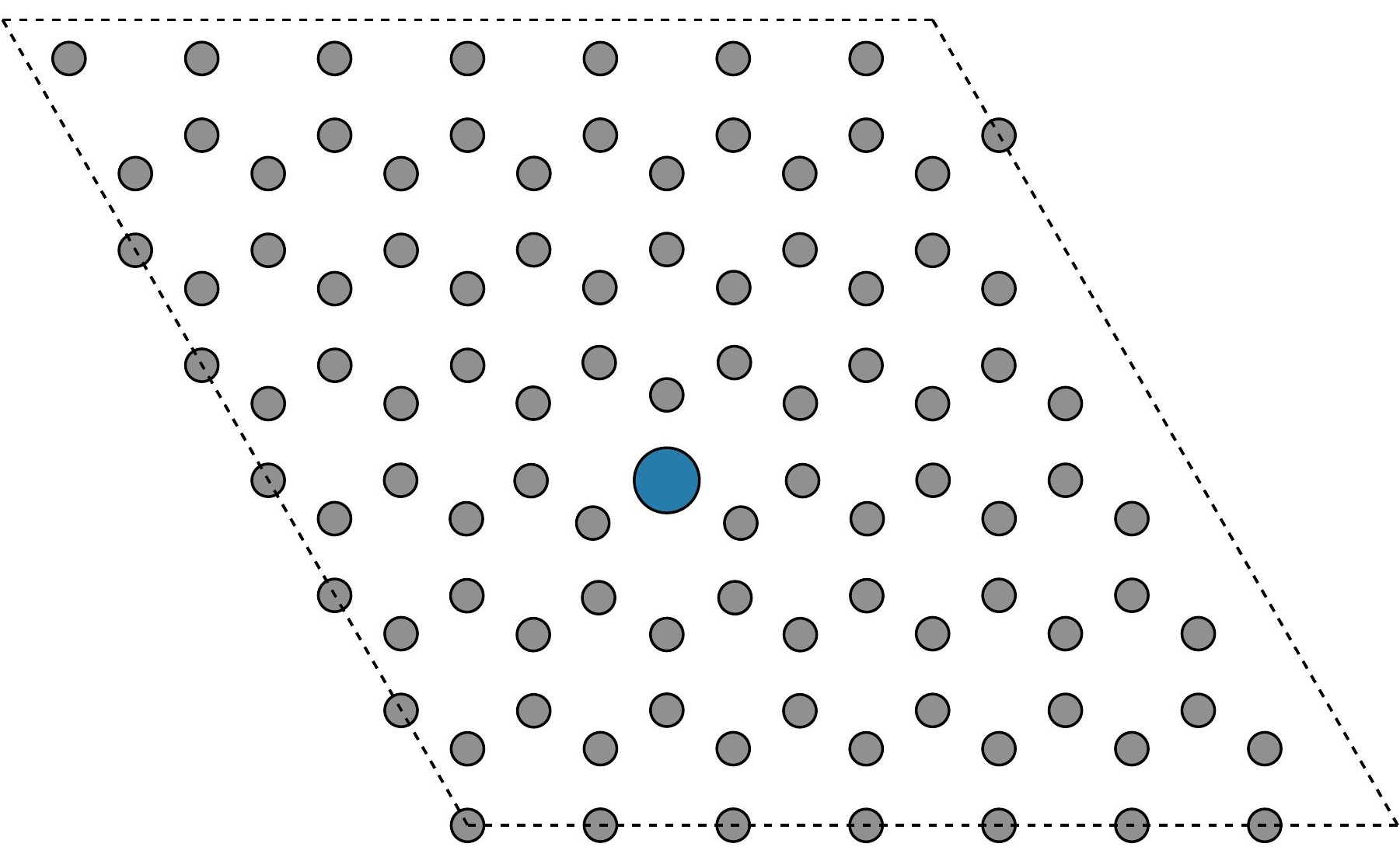


00000000880000000000

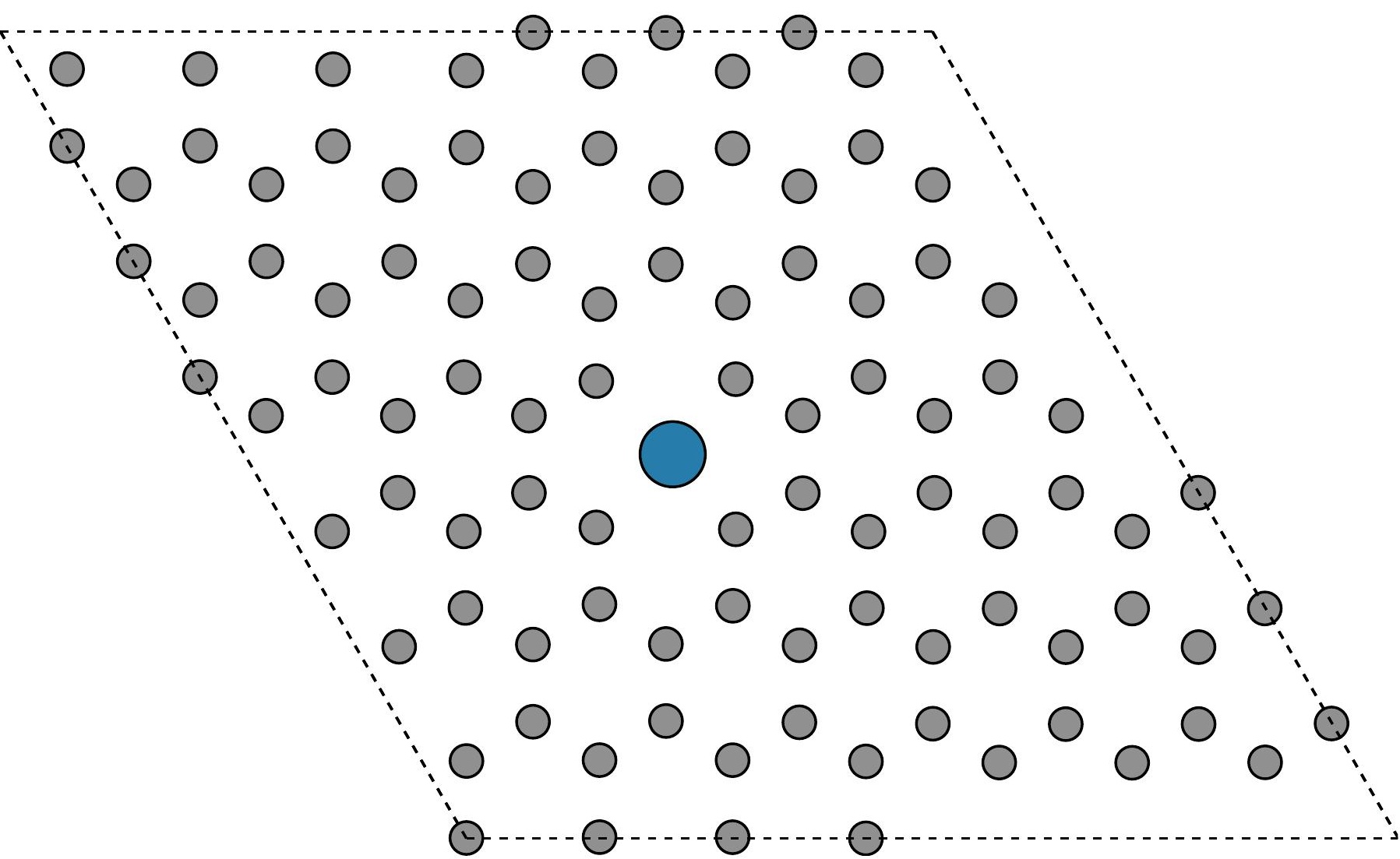


$+0.15 \mathrm{eV}$

. ० 00000890800000 ० ० ०

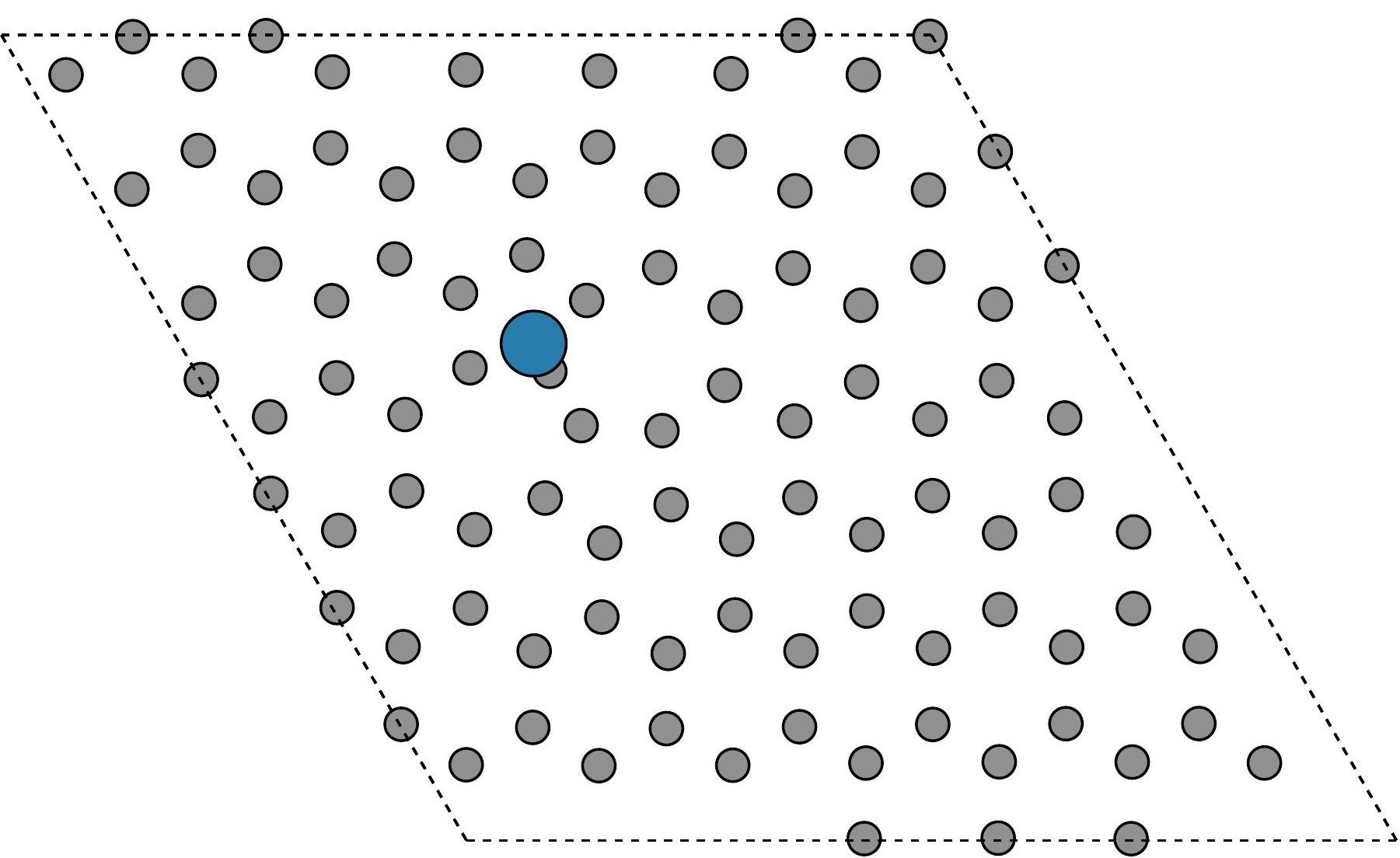




\section{$-0.61 \mathrm{eV}$}

- ค 8 8888888889800000

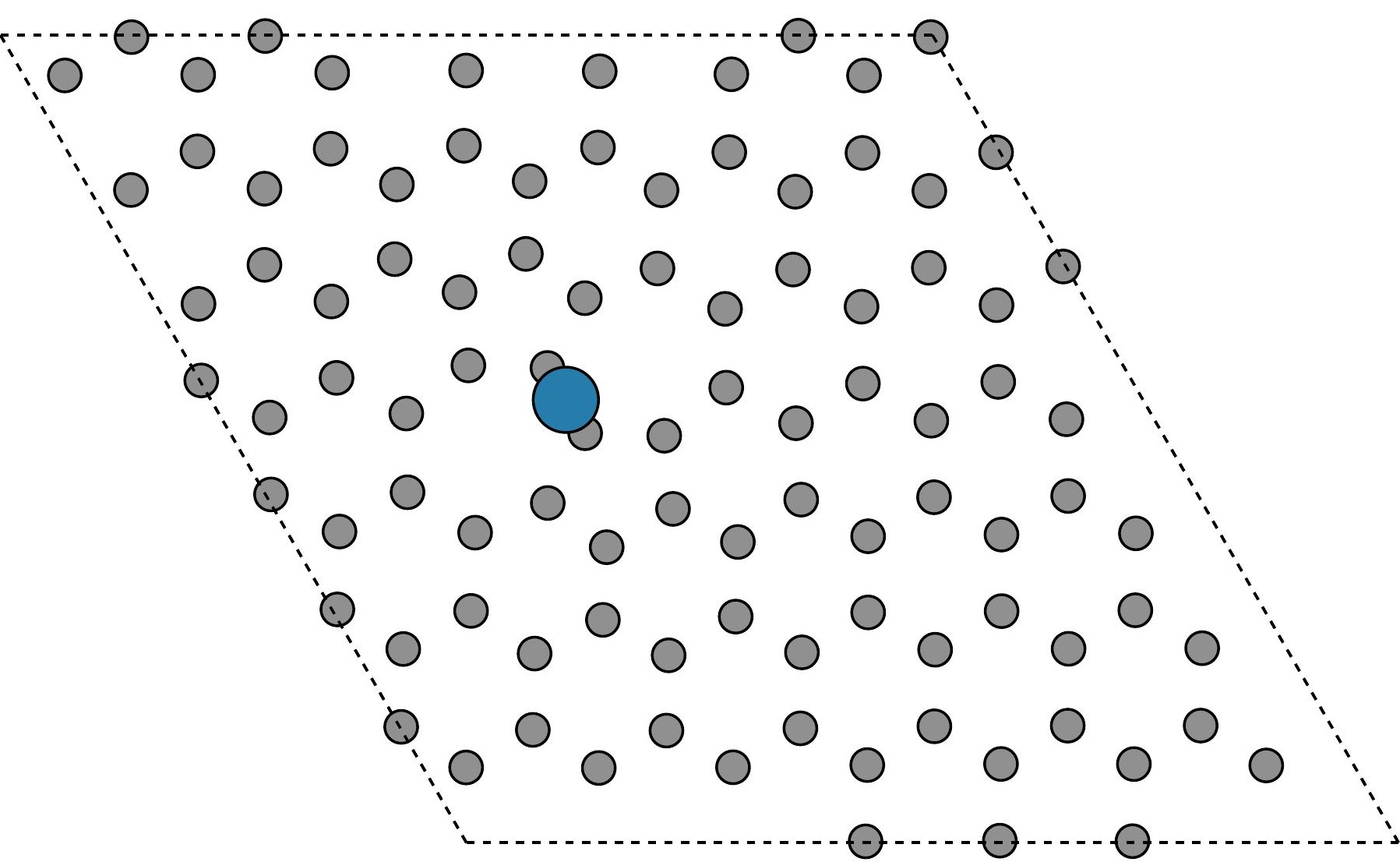

